\title{
INFLUÊNCIA DA RELAÇÃO ENTRE SÍNDROME METABÓLICA E PRÁTICA DE ATIVIDADE FÍSICA NA QUALIDADE DE VIDA DO INDIVÍDUO
}

\section{INFLUENCE OF THE RELATIONSHIP BETWEEN METABOLIC SYNDROME AND PRACTICE OF PHYSICAL ACTIVITY IN THE QUALITY OF LIFE OF THE INDIVIDUAL}

\author{
Acsa Quessada Gomes ${ }^{1}$, Débora Regina Hendges Poletto Pappen² \\ ${ }^{1}$ Nutricionista, Centro Universitário Fundação Assis Gurgacz (FAG). ${ }^{2}$ Mestre. Docente do Curso de \\ Nutrição, Centro Universitário Fundação Assis Gurgacz (FAG). \\ * Autor correspondente: de poletto@hotmail.com, https://orcid.org/0000-0003-0643-0346
}

\section{RESUMO}

Apesar de não haver um consenso sobre sua definição, a síndrome metabólica é um conjunto de hipertensão arterial, glicose elevada em jejum, dislipidemia e obesidade. Com isso, objetivou-se avaliar a relação entre patologia e prática ou não de atividade física de pacientes de um hospital de Cascavel-PR, propondo estratégias de prevenção e tratamento para melhora na qualidade de vida do paciente. Foi realizada a aplicação de um questionário para 23 pacientes entre 20 e 80 anos, escolhidos por meio de análise prévia dos prontuários. Desses pacientes, 9 eram mulheres e 14 homens. Entre as mulheres $33,3 \%$, e entre os homens $35,7 \%$ praticavam atividades físicas regularmente. As doenças que tiveram destaque foram diabetes mellitus e hipertensão, todavia, notou-se que os pacientes com hábito de prática de exercícios não apresentaram obesidade e dislipidemia. Ou seja, com as atividades físicas constantes há controle maior das doenças e prevenção de comorbidades. Por isso para melhora na qualidade de vida dos pacientes pode haver uma atuação entre diferentes profissionais da saúde incentivando a prática constante de exercícios e boa alimentação, oferecendo oportunidades como, por exemplo, a participação em grupos Hiperdia e mostras, relatando os pontos principais da síndrome metabólica e maneiras de prevenir e tratar.

Palavras chave: Síndrome metabólica. Pacientes hospitalizados. Atividade física.

\section{ABSTRACT}

Even though there is no agreement about its definition, metabolic syndrome is high blood pressure, high fasting glucose, dyslipidemia and obesity combined. This study aimed to evaluate the relationship between the pathology and practice or lack of physical activity of patients from a hospital in Cascavel-PR, offering prevention strategies and treatment to improve the pacient's life quality. A questionnaire was applied to 23 pacients between 20 and 80 years old, chosen by previous analysis of medical records. Out of these patients, 9 were women and 14 men. Among women $33,3 \%$, and $35,7 \%$ among men regularly exercise. The most common diseases were diabetes mellitus and hypertension; however, patients that exercised didn't show signs of obesity and dyslipidemia. That is, with the regular practice of exercises there is a greater control of diseases and prevention of comorbities. Therefore, in order to improve the patients' life quality, there could be the interaction between different health professionals encouraging the regular practice of exercises and good nutrition, offering 
opportunities, such as "Hiperdia" groups and exhibitions related to the disease, describing the main characteristics about metabolic syndrome and ways to prevent and treat it.

Keywords: metabolic syndrome, pacient's, physical activity

\section{INTRODUÇÃO}

A Síndrome Metabólica está relacionada a um conjunto de distúrbios metabólicos, ou seja, quando um paciente apresenta pressão arterial alta $(130 \mathrm{mmHg}$ e $85 \mathrm{mmHg}$ ) (MALACHIAS et al., 2016), nível de glicose em jejum elevado (100 mg/dL ou maior) (SOCIEDADE BRASILEIRA DE DIABETES, 2017), triglicerídeos $(150 \mathrm{mg} / \mathrm{dL}$ ou maior), HDL ( $<40 \mathrm{mg} / \mathrm{dL}$ em homens e $<50 \mathrm{mg} / \mathrm{dL}$ em mulheres) (XAVIER et al., 2013) e obesidade, este pode ser diagnosticado com a síndrome metabólica. Essas condições aumentam o risco de doenças cardiovasculares, diabetes e infarto (AMERICAN HEART ASSOCIATION, 2017).

Existe uma complexidade com respeito a síndrome metabólica e falta de consenso na definição da mesma, contudo, sabe-se que esta se tornou um problema global. Quanto mais cedo for realizado um diagnóstico e iniciar um tratamento, podese prevenir um aumento do risco de desencadear outros problemas, como por exemplo, câncer. Sendo assim, faz-se necessário, prevenção e tratamento adequado (O'NEILL; O'DRISCOLL, 2015).

O aparecimento da síndrome metabólica, não apenas em adultos, mas também em crianças e adolescentes, tem aumentado de forma acelerada, representando assim, um enorme risco para a população adulta futura. Algumas mudanças que afetaram no aumento da incidência deste problema na população são a falta de atividade física na maioria das pessoas, e um aumento das refeições fast food, ou seja, ricas em gordura saturada e sódio. Com isso, observa-se uma necessidade de medidas preventivas urgentes (NUTRITION AUSTRALIA, 2002).

As mudanças no estilo de vida, como atividade física, perda de peso, dieta saudável e equilibrada são alguns fatores que auxiliam na melhora e prevenção da síndrome metabólica (EBRAHIMOF; MIRMIRAN, 2013). O consumo excessivo de carboidratos se tornou um fator crítico no ganho de peso, obesidade e diabetes, entre outras, sendo essas as patologias relacionadas à síndrome metabólica. Desta maneira, faz-se necessário a adequação na dieta levando em consideração as patologias apresentadas. Em geral, recomenda-se trocar os carboidratos simples por complexos. A adição de fibras na dieta tem se destacado por estar associada à diminuição de diversos distúrbios metabólicos, como a hipertensão, diabetes, obesidade, doenças cardíacas e câncer de cólon. As gorduras saturadas promovem dislipidemias, sendo necessária uma diminuição desta e adequação das gorduras em geral (PITSAVOS et al., 2006).

A conduta mais adequada em casos de síndrome metabólica é trabalhar na modificação das causas principais: peso elevado, sedentarismo, procurando diminuir a resistência insulínica. Além de cuidar dos macronutrientes na dieta é necessário prestar atenção aos micronutrientes como o sódio, pois este afeta na sensibilidade à insulina, pressão arterial e aumento de calciúria. O magnésio atua no metabolismo de carboidratos, atuando de forma importante na homeostase de glicose e insulina. A perda de peso, aliada à atividade física auxilia na melhora das causas da síndrome. Uma dieta que tem se destacado é a mediterrânea por se mostrar benéfica no tratamento e prevenção por ser composta de cereais integrais, frutas, vegetais, gorduras monoinsaturadas prevalentes sobre as saturadas. Outra questão de 
destaque na síndrome metabólica é o estresse oxidativo. A alta produção de radicais livres está relacionada à ação insulínica, assim como os antioxidantes em baixas concentrações contribuem no risco de diabetes e patologias cardíacas; sendo assim, os portadores da síndrome em questão necessitam do consumo de frutas e vegetais por serem fontes de antioxidantes (SANTOS et al., 2006).

A prática regular de atividade física tem influência na incidência de riscos cardiovasculares, hipertensão, além de ajudar na diminuição da resistência à insulina, melhora o estado de dislipidemia, protege contra o desenvolvimento da síndrome metabólica. Ao relacionar os exercícios com a síndrome, observa-se que este auxilia na perda de peso e da gordura abdominal. Tanto os exercícios aeróbicos quanto os de resistência apresentam-se benéficos aos portadores da síndrome metabólica (EBRAHIMOF; MIRMIRAN, 2013).

Torna-se viável afirmar que a prática de atividade física regular pode beneficiar a saúde do indivíduo, além de atuar de forma preventiva em relação a doenças futuras. Sendo assim, um indivíduo que pratica atividade física desde cedo reduz as chances de apresentar alguma patologia mais tarde (SILVA; COSTA JR, 2011).

Levando em consideração o conceito e informações da patologia, a crescente expansão do problema e a importância da atividade física, objetivou-se uma avaliação da relação entre a patologia apresentada e a prática ou não de atividade física de pacientes que apresentavam hipertensão arterial, diabetes, obesidade ou dislipidemia de um hospital de Cascavel-PR, propondo, por meio da realidade local estratégias de prevenção e tratamento, visando o aprimoramento da qualidade de vida do paciente.

\section{METODOLOGIA}

Este estudo foi realizado em um hospital da cidade de Cascavel no Paraná com pacientes de ambos os gêneros, com idade entre 20 e 80 anos. Contando com a participação de 23 pacientes, não portadores de necessidades especiais, sem distinguir cor e raça, que estavam realizando tratamento e diagnosticados com síndrome metabólica e as patologias relacionadas. Para iniciarem o estudo, o projeto foi aprovado pelo Comitê de Ética e Pesquisa envolvendo seres humanos, com número de aprovação: 2.663.015. Além disso, os participantes assinaram um termo concordando em participar da pesquisa.

Para análise e avaliação entre a patologia apresentada e a prática de atividade física, foi realizado um questionário desenvolvido pela pesquisadora com os pacientes selecionados através dos prontuários previamente analisados. 0 questionário foi elaborado baseando-se em estudo relacionado à procura e desistência de atividade física em academia (MACHADO, 2016).

Após a realização do questionário e coleta de dados, foi realizado método de estatísticas em uma planilha no programa Microsoft Office Excel 2010.

\section{RESULTADOS E DISCUSSÕES}

Nesta pesquisa houve um total de 23 pacientes entrevistados, onde eram 9 mulheres e 14 homens. Sendo assim, a prevalência de síndrome metabólica fez-se presente em $60,8 \%$ dos homens e $39,1 \%$ das mulheres; sendo que destes, 8 praticavam atividade física e 15 eram sedentários, conforme representado na figura 1. 


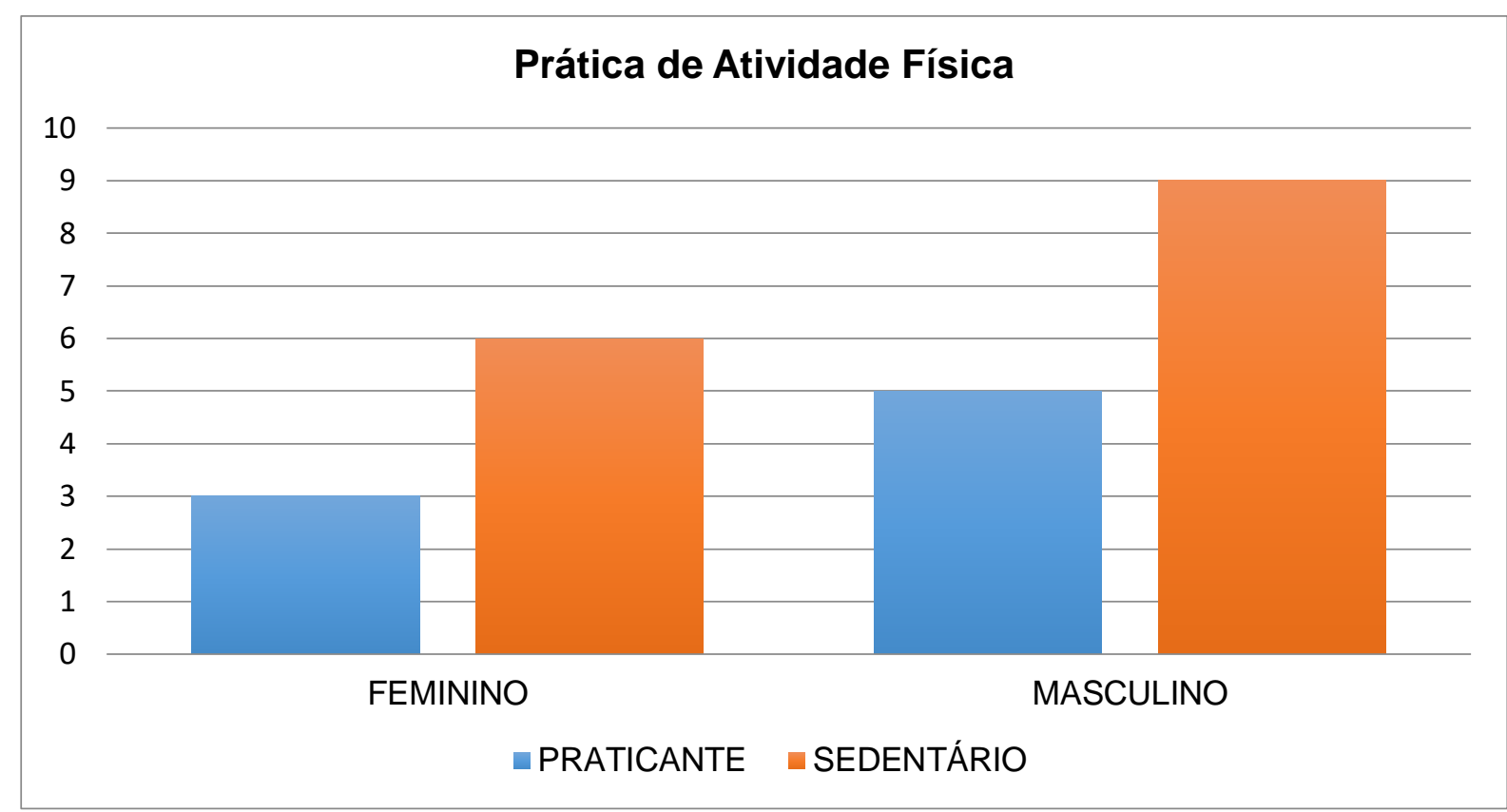

Figura 1. Relação entre pacientes e praticantes de atividade física ou sedentários

Por outro lado, ao analisar a prevalência mundial da síndrome metabólica, temse de acordo com a idade e o NCEP:ATP III (National Cholesterol Education Program's Adult Treatment Panel - Third adult treatment panel).

Tabela 01. Prevalência de síndrome metabólica mundial em homens e mulheres

\begin{tabular}{lll}
\hline PAÍS & HOMENS & MULHERES \\
\hline Austrália & $24,4 \%$ & $19,9 \%$ \\
China & $9,8 \%$ & $17,8 \%$ \\
Dinamarca & $18,6 \%$ & $14,3 \%$ \\
Índia & $17,1 \%$ & $19,4 \%$ \\
Irlanda & $21,8 \%$ & $21,5 \%$ \\
Coréia do Sul & $5,2 \%$ & $9,05 \%$ \\
Estados Unidos & $33,7 \%$ & $35,4 \%$ \\
\hline
\end{tabular}

Fonte: (O'NEILL; O'DRISCOLL, 2015).

Sendo que no Brasil observa-se:

Tabela 02. Prevalência de síndrome metabólica no Brasil

\begin{tabular}{lll}
\hline LOCAL & HOMENS & MULHERES \\
\hline SUS - Goiás & $55,7 \%$ & $60,5 \%$ \\
Ambulatório de Nutrição - Rio de Janeiro & $42,86 \%$ & $89,66 \%$ \\
\hline
\end{tabular}

Fonte: (VIEIRA; PEIXOTO; SILVEIRA, 2014; COSTA; VALLE, 2012).

Além desses, um estudo realizado em Vitória - Espírito Santo, envolvendo 1663 pessoas entre 25 e 64 anos observou-se prevalência de síndrome metabólica em $15,5 \%$ de pessoas entre 25 e 34 anos, e 48,3\% entre 55 e 64 anos (PENALVA, 2008).

Em geral, nota-se que houve prevalência da doença em pessoas do gênero feminino, embora na presente pesquisa a prevalência tenha sido em homens. 
Ao relacionar os participantes com as patologias especificas, 7 apresentaram diabetes, 2 obesidade, 2 dislipidemia e 21 hipertensão. Contudo, alguns pacientes apresentaram patologias associadas. Em diabetes, 3 dos pacientes eram mulheres e 4 homens; obesidade apresentou um paciente masculino e um feminino; dislipidemia apresentou 2 mulheres; e hipertensão teve sua maioria masculina, 13, e 8 mulheres. Nota-se na figura 2 que as doenças que mais se destacaram foram diabetes e hipertensão arterial.

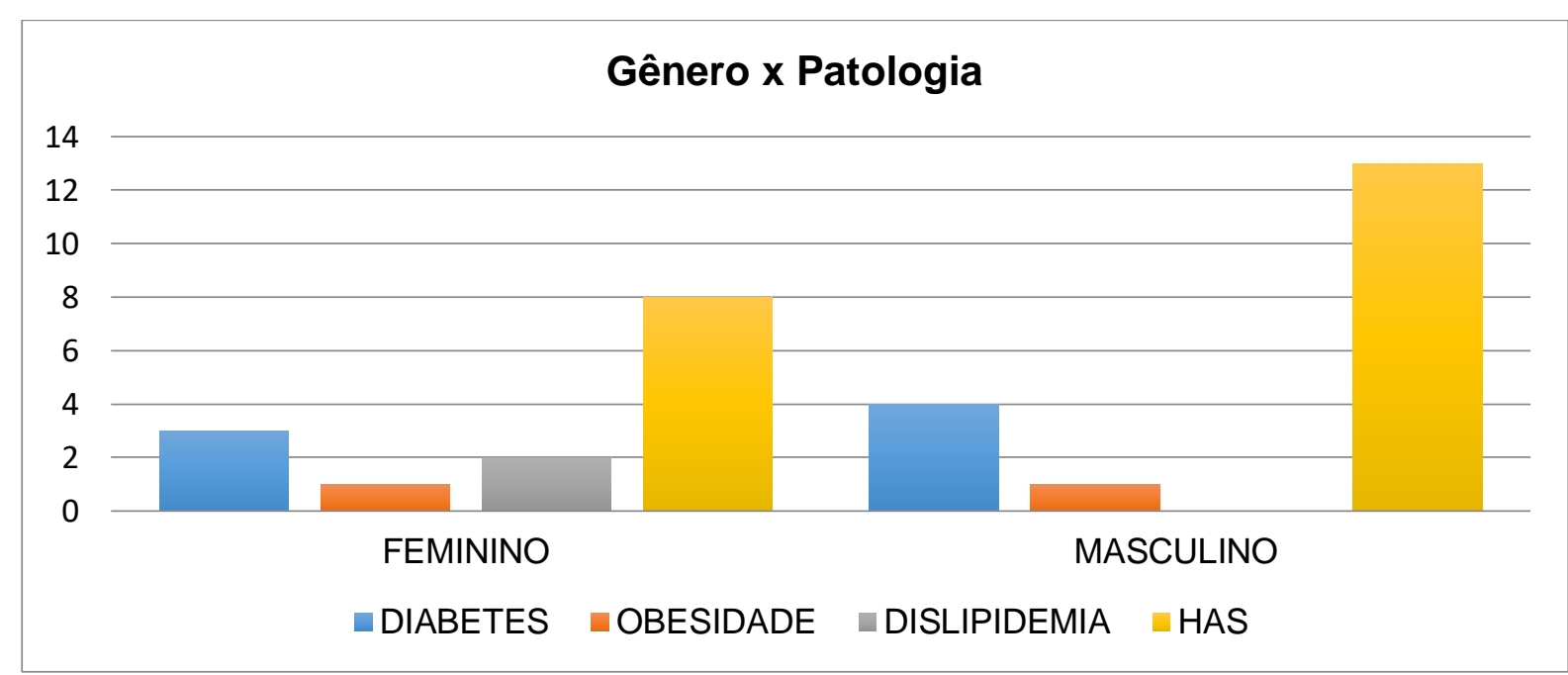

Figura 2. Relação entre gênero do paciente e a patologia apresentada.

Ao observar as patologias associadas à síndrome metabólica, um estudo realizado no Parque Indígena do Xingu que incluiu 170 indivíduos de ambos os sexos, com idade $\geq 20$ anos, com o intuito de verificar a existência da relação entre a atividade física e a presença da síndrome metabólica nos indivíduos do grupo, observa-se que do total, $40 \%$ eram mulheres, e a faixa etária foi em média entre 20 a 29 anos. Foi diagnosticada a síndrome metabólica em $27,8 \%$ dos participantes, sendo a maior parte mulheres. Ao subdividir nas patologias diagnosticadas na síndrome foi obtido: 6,8\% hipertensão, 23,1\% intolerância à glicose, 37,4\% hipertrigliceridemia, 43,5\% obesidade central, $66,2 \% \mathrm{HDL}-\mathrm{c}$ reduzido. A síndrome metabólica foi diagnosticada, em sua maior parte, em indivíduos $\geq 30$ anos; além de se tornar prevalente nos que apresentaram fraco ou regular desempenho nos teste de uma milha e também nos indivíduos classificados como sedentários ou pouco ativos. Sendo assim, concluiu-se que a falta da prática de atividade física e o baixo desempenho nos testes físicos estão relacionados com a maior incidência da síndrome estudada (SANTOS et al., 2012).

$\mathrm{Na}$ cidade de Alfenas, sul de Minas Gerais, em uma unidade de saúde foi avaliado 67 usuários adultos com diabetes mellitus, com o intuito de analisar a síndrome metabólica e a qualidade de vida dos pacientes. Destes, 59,7\% eram mulheres com idade média de 52,08 anos. Observou-se que do total avaliado 13,4\% eram tabagistas, 10,4\% faziam uso excessivo de álcool, $67,20 \%$ sedentários. Além do diabete mellitus, 58,2\% dos pacientes tinham hipertensão arterial associado. Do total de indivíduos, $43,58 \%$ estavam sobrepeso e $46,15 \%$ obesos. A prática regular de atividade física está relacionada com uma boa qualidade de vida, atuando na prevenção de doenças como diabetes e, consequentemente, a síndrome metabólica (MACCARONE et al., 2017).

Ao associar os resultados apresentados por Santos et al e Maccarone et al à pesquisa em questão, nota-se que as doenças que se destacam são a diabetes 
mellitus, hipertensão arterial e HDL-c reduzido; embora esta apresente uma amostragem menor.

No quesito de prática de atividade física apenas 8 de 23 eram praticantes, entre os quais 3 eram mulheres e 5 homens. Para analisar o motivo da prática de exercícios aplicou-se o questionário onde o paciente tinha as opções: emagrecimento, sair do sedentarismo e melhorar qualidade de vida, melhorar condicionamento físico, ganhar massa muscular, estética, indicação de médico/profissional da saúde, companhia amigos/família, hobbie. Alguns pacientes selecionaram mais de uma opção. Dentre estas, sair do sedentarismo e melhorar qualidade de vida (2), melhorar condicionamento físico (1) e hobbie (4) foram as opções que sobressaíram, conforme abaixo (Figura 3):

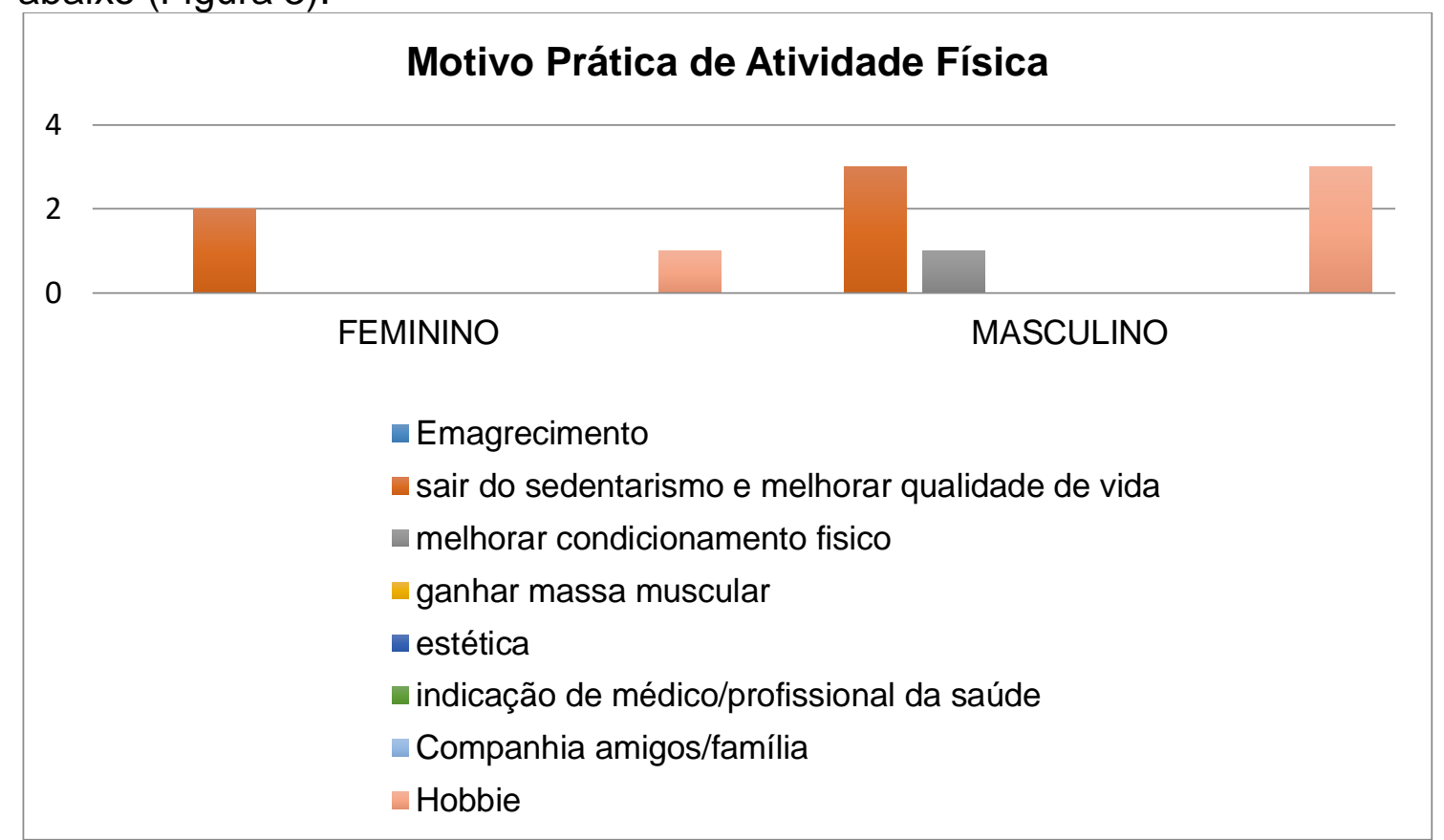

Figura 3: Motivo de prática de atividade física entre os pacientes fisicamente ativos de acordo com o gênero.

Já entre os pacientes sedentários, os motivos selecionados foram: falta de tempo (1), problemas de saúde (8), preguiça (5), outro (3), como ilustrados na figura 4. 


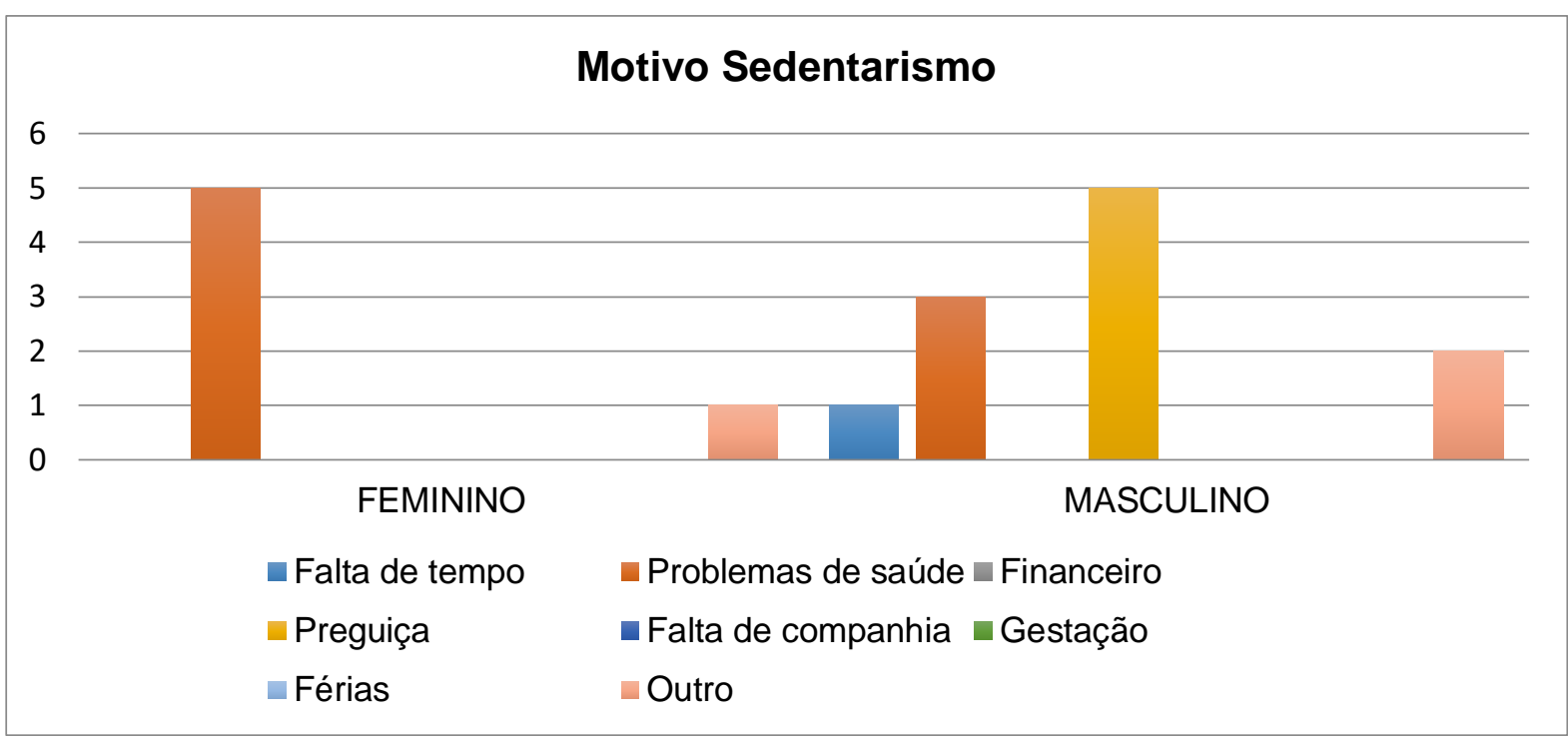

Figura 4. Motivo da falta de atividade física nos pacientes classificados como sedentários de acordo com o gênero

Com o intuito de melhorar a qualidade de vida, a melhor intervenção proposta pelo ATP III é a mudança da mesma, ou seja, perda de peso, prática de atividade física e hábitos alimentares saudáveis; sendo esta conduta considerada prioridade em relação ao tratamento com fármacos, pelo fato de os mesmos não melhorarem todos os sintomas apresentados pelos portadores da síndrome e suas patologias decorrentes, além de apresentarem efeitos colaterais e adversos (EBRAHIMOF; MIRMIRAN, 2013).

No estudo realizado na llha de São Miguel, Região Autônoma dos Açores Portugal, com amostra de 115 indivíduos caucasianos entre 22 e 54 anos de ambos os sexos, em que 68 eram mulheres e 47 homens. Sendo que 17,1\% dos homens apresentaram síndrome metabólica e 4,4\%, mulheres. Dentre estes observou-se que $70,7 \%$ apresentaram-se insuficientemente ativos em atividade física e 29,3\% ativos. Sendo que dos insuficientemente ativos na prática de atividade física 61,4\% apresentaram excesso de peso de acordo com o IMC e 69,2\% apresentaram obesidade. Contudo, nos ativos na prática de atividade física, 38,6\% apresentaram excesso de peso e 30,8\% obesidade. Em conclusão, observou-se que a obesidade é um problema quer seja isolado, ou também relacionado à síndrome metabólica e suas patologias. A atividade física, no entanto, pode contribuir na intervenção da patologia e como prevenção (SANTOS et al., 2005).

Dentro da categoria 'problemas de saúde' foram relatados: perna quebrada, cirurgia na coluna, doença de Chron, Alzheimer, dor nos ossos, uma perna amputada. Já em 'outros' foram: sedentarismo, o qual poderia ser considerado como a opção 'preguiça'; viagens a trabalho e por último, um paciente citou que por morar no sítio a locomoção para um lugar apropriado para praticar atividade física é difícil, porém relatou que sempre faz alguma coisa que movimente o corpo.

Entre os 8 praticantes de atividade física, as patologias encontradas foram diabetes mellitus e hipertensão arterial conforme tabela abaixo (Tabela 03). Além disso, pode-se observar que com a prática de atividade física não há relato de obesidade e dislipidemia, o que pode ser consequência do tamanho da amostra. 
Tabela 03. Relação praticantes de atividade física e patologia apresentada

\begin{tabular}{lll}
\hline PRATICANTES & DM & HAS \\
\hline FEMININO & 1 & 2 \\
MASCULINO & 0 & 5 \\
\hline
\end{tabular}

No que diz respeito ao efeito da prática de atividade física na pressão arterial, um ensaio relacionando comportamento da pressão arterial e composição corporal de participantes com síndrome metabólica, onde 49 voluntários foram avaliados sendo dividido, randomicamente, em dois grupos TAI (treinamento aeróbio intervalado) e TRC (treinamento resistido convencional), além do grupo controle, tendo cada grupo 15 indivíduos. Observou-se que uma diminuição média de $7,77 \mathrm{mmHg}$ na pressão sistólica do grupo TAl após o treinamento, resultando em influência do mesmo na pressão sistólica em adultos sedentários, constatando em melhora. No entanto, no grupo TRC não houve mudanças na pressão arterial. No IMC constatou-se aumento no grupo controle e redução no grupo TAl. Concluindo assim, que ambos os treinamentos não diminuíram significativamente a pressão arterial, mesmo que o TAI exercesse maior influência na redução da pressão arterial (SILVA et al., 2017).

\section{CONSIDERAÇÕES FINAIS}

Ao observar a pesquisa realizada não foi possível chegar a uma conclusão específica pelo fato do número da amostra ser pequeno em comparação a outras pesquisas, porém pode ser dada continuidade na pesquisa e questionário com os pacientes para observar se há uma resposta diferente e mais acurada. Contudo, analisando os dados apresentados por diferentes autores, nota-se uma relação entre as patologias e a prática de atividade física ou a falta da mesma. Com a prática de atividade física há um controle maior da doença e uma prevenção de outras patologias.

Entre os pacientes participantes desta pesquisa houve apenas um que se destacou pela idade jovem, sendo que os demais encontram-se na terceira idade. A paciente jovem pratica atividade física, e a maioria dos pacientes não tem esse hábito. Quanto mais cedo houver uma conscientização sobre prática de atividade física relacionada a uma boa qualidade de vida, o paciente poderá usufruir de benefícios em longo prazo.

Assim sendo, pode haver uma atuação conjunta multidisciplinar entre médicos, enfermeiros e nutricionistas, no incentivo à prática de atividade física aliada a uma boa alimentação, melhorando a qualidade de vida do paciente. Pelo fato da maioria dos pacientes serem hipertensos e diabéticos, os profissionais do hospital podem indicálos ao grupo Hiperdia realizado nas Unidades Básicas de Saúde dos bairros, sendo o mesmo de acesso livre e gratuito, porém com informações válidas e que agregam conhecimento auxiliando na melhora do paciente. Junto a isso, pode ser realizada uma mostra relatando os problemas da síndrome metabólica e suas patologias relacionadas, além de apresentar a prevenção e tratamento, focando na mudança de hábitos alimentares, na qual poderá ser distribuído panfletos com dicas de pratos saudáveis, práticos e saborosos, enfatizando a prática de atividade física fazendo aquilo que o paciente realmente gosta, seja caminhada, corrida, pilates, academia, natação, hidroginástica, outros. 
É importante levar em consideração que tanto para a prevenção quanto tratamento é preciso uma mudança definitiva no estilo e qualidade de vida, cuidando e promovendo a saúde do indivíduo.

\section{REFERÊNCIAS}

AMERICAN HEART ASSOCIATION. What is Metabolic Syndrome? Disponível em: <http://www.heart.org./HEARTORG/Conditions/More/MetabolicSyndrome/AboutMetabolic-Syndrome_UCM_301920_Article.jsp\#Wyg9Qy3OqT8 >. Acesso em: 10 de Março de 2018.

COSTA, M.F.; VALLE, J.Do. Síndrome Metabólica: Prevalência e Associação com Doenças Cardiovasculares em Adultos. DEMETRA: Alimentação, Nutrição \& Saúde, v.7, n. 2, p. 119-132, 2012.

EBRAHIMOF, S.; MIRMIRAN, P. Nutritional Approaches for Prevantion and Treatment of Metabolic Syndrome in Adults. Journal of Paramedical Sciences, v. 4, n. 2, p. 123134, 2013.

MACCARONE, S.D.; LIMA, D.B.; FERREIRA, E.B. Rastreamento da Síndrome Metabólica e Qualidade de Vida dos Diabéticos Adscritos a uma Unidade de Estratégia de Saúde da Família em um Município do Sul de Minas Gerais. Revista de Atenção à Saúde, v. 15, n. 51, p. 98-105, 2017.

MACHADO, C.V. Motivos da Procura e Desistência da Prática de Atividade Física em uma Academia de Santa Rosa-RS. 2016. 36 f. Dep. de Ciências Administrativas, Contábeis Econômicas da Comunicação Curso de Pós-Graduação Lato Sensu MBA em Gestão Empresarial. Universidade Regional do Noroeste do Estado do RS, Rio Grande do Sul.

MALACHIAS M.V.B.; SOUZA W.K.S.B.; PLAVNIK F.L. et al. 7ª Diretriz Brasileira de Hipertensão Arterial. Arquivos Brasileiros de Cardiologia, v. 107, n. 3, s. 3, 2016.

NUTRITION AUSTRALIA. Nutrition Fact Sheet What does 'Metabolic Syndrome' mean? How common is the Metabolic Syndrome? What are the Health Implications of Having Metabolic Syndrome? What can be done to reduce my risk of developing Metabolic Syndrome, or to help overcome it, p. 1-6, 2002.

O'NEILL, S.; O'DRISCOLL, L. Metabolic Syndrome: a closer look at the growing epidemic and its associated pathologies. Obesity Reviews, v. 16, n. 1, p. 1-12, 2015.

PENALVA, D.Q.F. Síndrome metabólica: diagnóstico e tratamento. Revista de Medicina, v. 87, n. 4, p. 245, 2008.

PITSAVOS, C. et al. Diet, exercise and the metabolic syndrome. The Review of Diabetic Studies: RDS, v. 3, n. 3, p. 118-26, 2006.

SANTOS, C.R.B. et al. Fatores Dietéticos na Prevenção e Tratamento de Comorbidades Associadas à Síndrome Metabólica. Revista de Nutrição, v. 19, n. 3, p. 389-401, 2006. 
SANTOS, K.M. et al. Grau de Atividade Física e Síndrome Metabólica: um estudo transversal com indígenas Khisêdjê do Parque Indígena do Xingu, Brasil. Caderno de Saúde Pública, v. 28, n. 12, p. 2327-2338, 2012.

SANTOS, R.; NUNES, A.; RIBEIRO, J.C.; SANTOS, P.; DUARTE, J.A.R.; MOTA, J. Obesidade, Síndrome Metabólica e Atividade Física: estudo exploratório realizado com adultos de ambos os sexos, da llha de S. Miguel, Região Autônoma dos Açores, Portugal. Revista Brasileira de Educação Física e Esporte, v. 19, n. 4, p. 317-328, 2005.

SILVA, H.; CASTRILLÓN, C.; FIGUEIREDO, M.; VANDERLEI, F.; LINARES, S.; LEMOS, L.; HIDALGO, R.; MILANEZ, A.; BATISTA, N.; PASTRE, C.; NETTO, JUNIOR, J. Comportamento da Pressão Arterial e Composição Corporal Após dois tipos de Tratamento Periodizados em participantes com Síndrome Metabólica. Ensaio Clínico Controlado Randomizado. Colloquium Vitae, v. 9, n. 1, p. 28-33, 2017.

SILVA, P.V.C.; COSTA JR, A.L. Efeitos da Atividade Física para a Saúde de Crianças e Adolescentes. Psicologia Argumento, v. 29, n. 4, p. 805-817, 2014.

SOCIEDADE BRASILEIRA DE DIABETES. Diretrizes da Sociedade Brasileira de Diabetes 2017-2018. São Paulo, 2017.

VIEIRA, E.C.; PEIXOTO, M.do.R.G.; SILVEIRA, E.A.da. Prevalence and Factors associated with Metabolic Syndrome in elderly users of the Unified Health System. Revista Brasileira de Epidemiologia, v. 17, n. 4, p. 805-8017, 2014.

XAVIER H.T.; IZAR M.C.; FARIA NETO J.R.; ASSAD M.H.; ROCHA V.Z.; SPOSITO A.C.; FONSECA F.A.; dos SANTOS J.E.; SANTOS R.D.; BERTOLAMI M.C.; FALUDI A.A.; MARTINEZ T.L.R.; DIAMENT J.; GUIMARÃES A.; FORTI N. A.; MORIGUCHI E.; CHAGAS A.C.P.; COELHO O.R.; RAMIRES J.A.F. V Diretriz Brasileira de Dislipidemias e Prevenção da Aterosclerose. Arquivos Brasileiros de Cardiologia, v. 101, n. 4, s. 1, 2013. 